\title{
A Powerful Remote Source of O Atoms for the Removal of Hydrogenated Carbon Deposits
}

\author{
R. Zaplotnik $\cdot$ A. Vesel $\cdot$ M. Mozetic
}

Published online: 21 March 2012

(C) The Author(s) 2012. This article is published with open access at Springerlink.com

\begin{abstract}
We describe a system to deliver a large flux of $\mathrm{O}$ atoms for the removal of hydrogenated carbon films from surfaces in remote areas of tokamaks with carbon divertors. The oxygen plasma is generated via electrodeless radiofrequency discharge in a discharge chamber connected to a remote chamber by a $2 \mathrm{~m}$ long complexshaped glass tube $4 \mathrm{~cm}$ in diameter. The density of $\mathrm{O}$ atoms in the remote chamber was measured with a nickel catalytic probe and its variation with discharge power obtained. The density was close to the detection limit of the probe (around $1 \times 10^{19} \mathrm{~m}^{-3}$ ) as long as the vacuum system was pumped with a rotary pump at a nominal pumping speed of $80 \mathrm{~m}^{3} \mathrm{~h}^{-1}$. The density increased well over $10^{20} \mathrm{~m}^{-3}$ when a Roots pump was added. The effective pumping speed at the current setup was up to $200 \mathrm{~m}^{3} \mathrm{~h}^{-1}$. At such conditions, the maximal $\mathrm{O}$-atom density at $2 \mathrm{~m}$ from the source was up to $3 \times 10^{20} \mathrm{~m}^{-3}$. The density depended on the pressure as well as the discharge power. The behavior of O-atom density far away from the source was explained by gas phase and surface phenomena. The effective pumping speed was found to be of crucial importance. The setup was used for removal of model hydrogenated carbon films. Experiments were performed at sample temperatures up to $600 \mathrm{~K}$ and etching rates up to $50 \mathrm{~nm} / \mathrm{s}$ were obtained. We found that the experimental setup is suitable for removal of hydrogenated atoms on a large scale.
\end{abstract}

Keywords Oxygen plasma - Source of neutral oxygen atoms - O-atom density - Hydrogenated carbon removal . Cleaning tokamak divertors

R. Zaplotnik ( $₫) \cdot$ A. Vesel · M. Mozetic

Jožef Stefan Institute, Jamova 39, 1000 Ljubljana, Slovenia

e-mail: rokzaplotnik@yahoo.com

\section{Introduction}

As a promising energy source for future generations, nuclear fusion of heavy hydrogen isotopes still attracts interest. Fusion is obtained by different means but the most promising is from magnetically-confined high-temperature hydrogen plasmas. Magnetic confinement enables high plasma temperatures to be sustained as well as reasonable discharge duration. Several confinement configurations have been applied including large tokamaks. In such devices, plasma-facing components are subject to extreme conditions from hot gaseous particles interacting with solid material. The most severe conditions are found in the divertors where the power density is of order $10 \mathrm{MW} \mathrm{m}^{-2}$. The divertor materials must withstand such conditions to ensure proper operations. Various materials have been tried in the construction of the first wall blankets; currently carbon and tungsten seem to be the most suitable. Both materials have, however, disadvantages; tungsten, a high- $Z$ material, causes substantial plasma cooling in the discharge volume even in small concentrations, whereas carbon suffers from chemical sputtering. The resultant $\mathrm{CH}_{x}$ radicals stick to the surfaces in remote parts of a tokamak causing the formation of hydrogenated carbon deposits. Not only does chemical sputtering cause erosion of the first wall material, and thus modification of the divertor components dimensions, but also retains radioactive tritium, thereby creating a bigger problem [1]. Although, thermomechanical properties of carbon fiber composites (CFCs) are superior in comparison to tungsten, the interaction of high-energy neutrons with carbon materials causes radiation damage, localized amorphization, and thus a loss of otherwise excellent thermomechanical properties. Nowadays, it is believed that, although CFCs have remained the material to form the blankets of the most exposed parts of 
divertors in the initial operation stage of the international experimental fusion reactor ITER, these will not be used in commercial fusion devices.

Since chemical erosion of carbon cannot be avoided, many researchers worldwide are currently occupied with the development of techniques to suppress carbon hydrogenation. A promising technique to minimize $\mathrm{H}-\mathrm{C}$ deposition is puffing nitrogen-containing gases into recesses in the divertor. Recent experiments showed that the technique allows for the important reduction of $\mathrm{H}-\mathrm{C}$ depositions through the molecular formation of $\mathrm{HCN}$, which by not sticking to the walls are easily pumped out [2-4]. Scavenger techniques, however, do not prevent deposition completely so researchers have developed techniques for the occasional removal of these deposits. Carbon deposits can be removed with different techniques, e.g. with pulsedlaser [5] or with oxidation, which can be divided into three categories: plasma driven, activated oxygen and thermooxidation [6-8]. Applications of mild plasma of oxygen, hydrogen, or nitrogen (ammonia) were found suitable [915] but this technique cannot be applied in ITER because of the strong permanent magnetic fields produced by superconducting magnets. Nonetheless, the erosion rates for hydrogenated carbon deposits were found suitable $\sim 2.1 \times 10^{19} \mathrm{C} / \mathrm{s}$ [14]. Also, it was found that the probability for the removal of a carbon by a positively-charged oxygen ion is well over $100 \%$. That paradox has been explained as the removal of $\mathrm{C}$ atoms from hydrogenated carbon films by other reactive particles found in the oxygen plasma. The primary candidates are neutral oxygen atoms.

Neutral oxygen atoms can be produced in different oxygen plasma discharges. Average neutral oxygen atom densities measured in these discharges are: $\sim 1 \times 10^{21} \mathrm{~m}^{-3}$ in a DC discharge [16], $\sim 5 \times 10^{20} \mathrm{~m}^{-3}$ in a microwave discharge [17], $\sim 1 \times 10^{20} \mathrm{~m}^{-3}$ in a capacitively coupled $\mathrm{RF}$ plasma (CCP) [18], $\sim 2 \times 10^{21} \mathrm{~m}^{-3}$ in an oxygen plasma created by electrode-less high-frequency discharges (ICP) [19-25]. In inductively coupled plasmas, because of relatively-low dissociation energies for oxygen molecules as compared with their ionization energy, and due to a relatively low probability for recombination on chamber walls, the dissociation fraction in plasma created in such discharges easily exceed $10 \%$. This is orders of magnitude larger than the ionization fraction in mild plasmas. The huge difference between the dissociation and ionization fractions has been attributed to the minimal loss of $\mathrm{O}$ atoms on surfaces; the recombination coefficient depends on the type of material facing the plasma and can be as low as $10^{-5}$ for some glasses [26-29]. The probability for surface neutralization of charged particles, in contrast, is close to 1 .

High chemical reactivity of neutral oxygen atoms and ease of production have led to various experiments on the removal of hydrogenated carbon deposits by interaction with atoms but only with the absence of all other plasma particles. First results showed that the reaction probability could be appreciatively high at elevated temperatures. Removal rates exceeding $10 \mathrm{~nm} \mathrm{~s}^{-1}$ have been reported $[30,31]$. The cited results have been obtained in small experimental systems with typical sample size of the order of $\mathrm{cm}^{2}$. The problem with this technique, as with most others, however, is in scaling up the experimental conditions so that the method can be used in the removal of deposits in large fusion reactors. Total surface areas of the order of $100 \mathrm{~m}^{2}$ are typical.

Removal of hydrogenated carbon deposits with neutral oxygen atoms over large areas requires the development of a huge source of $\mathrm{O}$ atoms as well as techniques for transferring atoms from discharge to those recess areas. As mentioned earlier, the source cannot be placed inside the main tokamak vessel; the closest suitable place is about $2 \mathrm{~m}$ away from the large tokamak divertor. In transfer, atoms can be lost because of recombination hence the $\mathrm{O}$-atom density in the interior of the divertor will be much lower than in the discharge area itself. To show the viability of this technique in removing deposits from recesses, we developed a large $\mathrm{O}$ atom source and delivery system comprising a reaction chamber attached with a rather complex-shaped tube $2 \mathrm{~m}$ in length. The complex shape is to simulate possible access routes to the recesses in the divertor. Details and tests of the setup are presented in this paper.

\section{Experimental Setup}

The source of the neutral oxygen atoms is from electrodeless radiofrequency discharges. The discharge chamber is a $2 \mathrm{~m}$ long, $20 \mathrm{~cm}$ wide glass tube. Commercially available oxygen is diffused into the discharge chamber from one end through a flow controller. At the other end, the discharge chamber is furnished with an aluminum flange and connected to a remote chamber by a glass tube with an inner diameter of $3.4 \mathrm{~cm}$ and a length of $2 \mathrm{~m}$. A schematic of the experimental setup is presented in Fig. 1 with an inset detailing the shape of the connection tube. This complicated shape was chosen because we wanted to address issues concerning possible routing access difficulties to the divertors. The remote chamber is a Pyrex tube with diameter of $30 \mathrm{~cm}$ and length of $27 \mathrm{~cm}$. It is pumped with a Roots pump with a nominal pumping speed of $400 \mathrm{~m}^{3} \mathrm{~h}^{-1}$ backed by a two-stage oil rotary pump with a pumping speed of $80 \mathrm{~m}^{3} \mathrm{~h}^{-1}$. The system is equipped with several absolute vacuum gauges covering the range from 1 to $1,000 \mathrm{~Pa}$, and two different catalytic probes for measurement of the density of $\mathrm{O}$ atoms at both ends of the connection tube. 


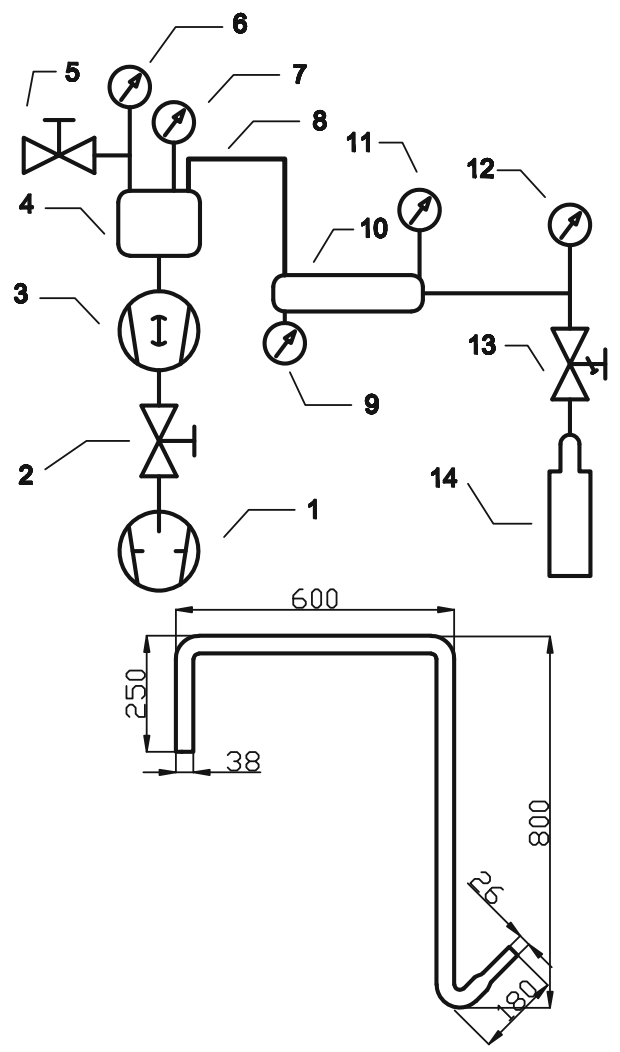

Fig. 1 The experimental setup. 1 Rotary pump, 2 gate valve, 3 roots pump, 4 remote chamber, 5 inlet valve for venting, 6 catalytic probe in remote chamber, 7 pressure gauge in remote chamber, 8 connecting glass tube, 9 catalytic probe at the edge of the discharge chamber, 10 discharge chamber, 11 pressure gauge in discharge chamber, 12 vacuum gauge, 13 flow controller, 14 oxygen flask. The inserted figure shows the exact dimensions of the connection tube

Plasma is generated inside the discharge tube, which is mounted with a couple of parallel coils that are connected to a radiofrequency (RF) generator. The RF generator is optimized for electrode-less coupling and operates at 27.12 MHz frequency. The output power, with the nominal power of $10 \mathrm{~kW}$, is adjustable. The parallel coupling of the RF coils presented in Fig. 2 allows for almost optimal uniformity of plasma inside the discharge chamber and a rather low peak voltage that helps keeping stray effects tolerable. A heatable sample holder is placed into the remote chamber for quantification of results on removal of hydrogenated carbon films by neutral oxygen atoms.

\section{Results}

\section{Vacuum Parameters}

The rather large volume of the experimental setup is pumped by the Roots and/or rotary pumps. The pressure in the discharge and remote chambers is measured during

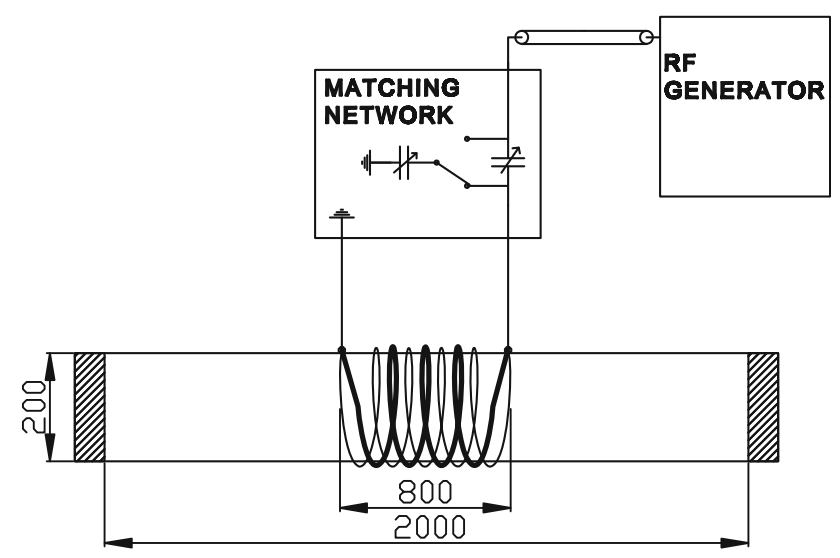

Fig. 2 The coupling between the RF generator and plasma

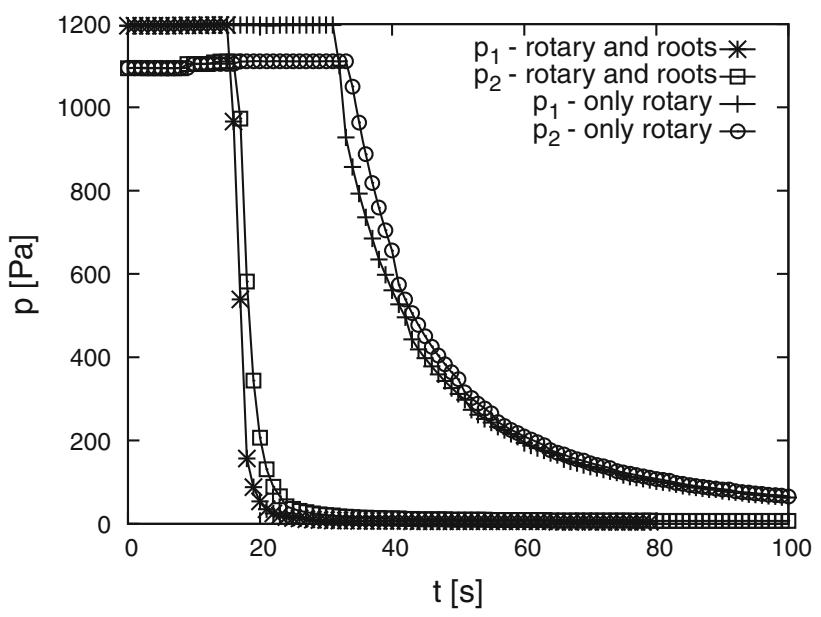

Fig. 3 Pressure in the remote chamber $p_{1}$ and in the discharge chamber $p_{2}$, pumped with roots and rotary pump (left two curves) and only with rotary pump (right two curves), versus pumping time. The pumps were turned on at $t=0 \mathrm{~s}$

evacuation and a typical result is shown in Fig. 3. Because of range limitations in the gauges, the pressure becomes measurable only after about $20 \mathrm{~s}$ (if the system is pumped with both pumps) or about $40 \mathrm{~s}$ (if only the rotary pump is used). The pressure in the remote chamber drops faster than in the discharge chamber. This effect is explained by a limited conductance of the connection tube. The curves presented in Fig. 3 allow for a calculation of the effective pumping speed at the remote chamber. The effective pumping speed is calculated using the standard vacuum equation

$S_{\text {eff }}=\frac{-V \cdot d p / d t}{p-p_{u}}$.

Here, $d p / d t$ is the pressure derivative measured in the remote chamber, $V$ is the total volume of the vacuum system, $p$ is the pressure measured in the remote chamber, and $p_{u}$ is the ultimate pressure achieved in the remote 


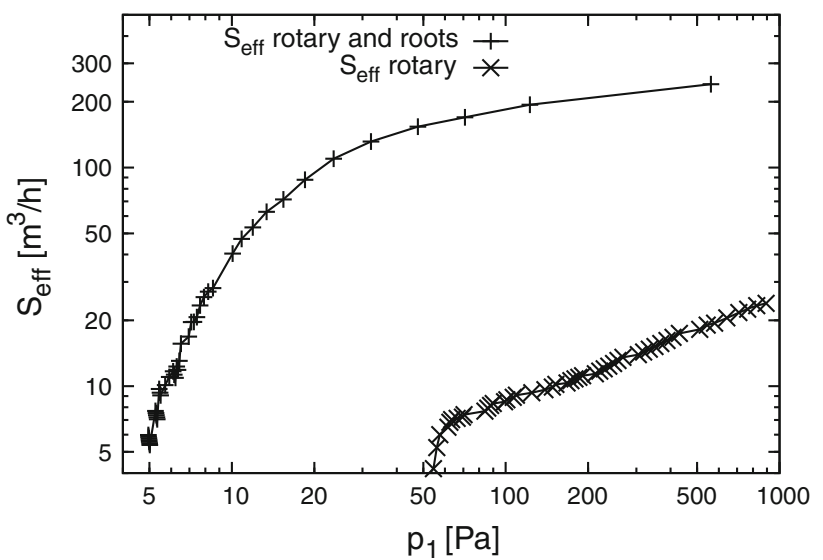

Fig. 4 The effective pumping speed versus pressure in the remote chamber. The effective pumping speed is calculated from pumping curves. The lower curve is for the case the system is pumped with rotary pump only while the upper one is for combination of rotary and roots pumps

chamber after about an hour of pumping. The effective pumping speed versus the pressure in the remote chamber as calculated from Eq. (1) is plotted in Fig. 4. A rather poor pumping speed as determined from (1) illustrates the influence of the rather narrow and long connecting tube between the discharge and remote chambers. Under continuous supply of oxygen, the effective pumping speed is different and can be calculated using the continuity equation

$S_{\text {eff }}=S_{M F C} \cdot p_{S} / p_{1}$.

Here, $S_{M F C}$ is the volume flow rate through mass flow controller at standard pressure $p_{S}$ and $p_{1}$ is pressure in the remote chamber. The effective pumping speed using Eq. (2) is plotted versus the pressure in the remote chamber in Fig. 5. Values are much higher than for those calculated from measured pumping curves using Eq. (1). The

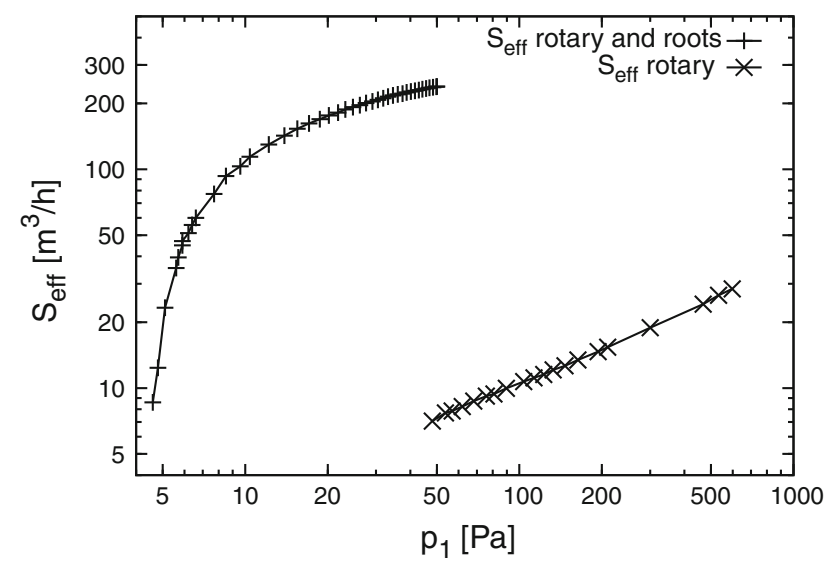

Fig. 5 The effective pumping speed as calculated using continuity equation importance of the pumping speed will be demonstrated in a later section.

Understanding the effective pumping speeds enables the determination of the conductance of the connection tube. The conductance is

$C_{12}=p_{1} S_{\text {eff }} /\left(p_{2}-p_{1}\right)$.

Here, $p_{1}$ and $p_{2}$ are the respective pressures in the remote and discharge chambers and $S_{\text {eff }}$ is the effective pumping speed calculated with Eq. (3). The lower curve in Fig. 6 is a plot of the conductance for the connection tube versus the average pressure in the remote and discharge chambers. The conductance can be also calculated using the appropriate approximation from standard vacuum literature [32]:

$$
\begin{aligned}
C_{12}(\bar{p})= & 0.91 \cdot\left(4.86 \cdot \frac{d^{4}}{l} \cdot \bar{p} \cdot \frac{\mathrm{m}^{3}}{\mathrm{~cm}^{3} \mathrm{Pah}}\right. \\
& \left.+43.56 \cdot \frac{d^{3}}{l} \cdot \frac{1 \mathrm{~cm} \mathrm{~Pa}+1.92 \cdot d \cdot \bar{p}}{1 \mathrm{~cm} \mathrm{~Pa}+2.37 \cdot d \cdot \bar{p}} \cdot \frac{\mathrm{m}^{3}}{\mathrm{~cm}^{3} \mathrm{Pah}}\right) .
\end{aligned}
$$

Here, $d$ is the inner diameter of a tube in $\mathrm{cm}, l$ is the tube length in $\mathrm{cm}$, and $\bar{p}$ is the average pressure between the ends of a tube in Pa. The conductance calculated according to Eq. (4) is represented in Fig. 6 with the upper curve. The discrepancy is within expectations, the model being just the first approximation. More important is the fact that the conductance is about the same as the effective pumping speed.

\section{Catalytic Probe Results}

Systematic measurements of the catalytic probe were performed after measuring the vacuum parameters. The steady temperature of the catalytic probe positioned inside the

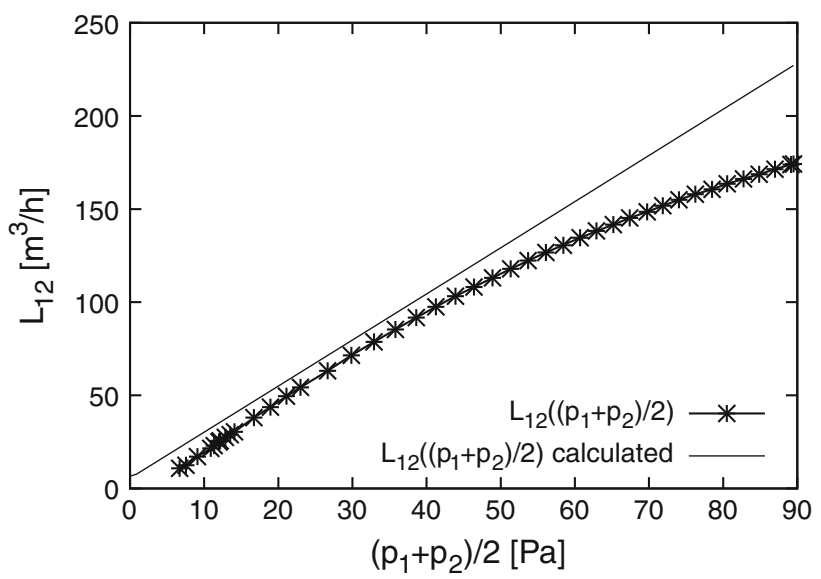

Fig. 6 The conductance of the connecting tube 


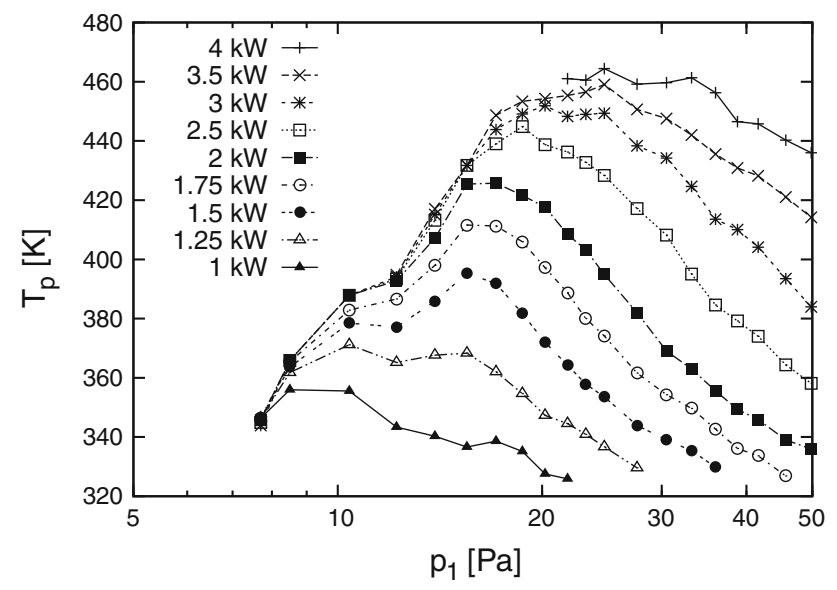

Fig. 7 The catalytic probe temperature versus pressure in the remote chamber

remote chamber was measured versus pressure at different discharge power. The results are summarized in Fig. 7. The parameter is the discharge power.

The density of $\mathrm{O}$ atoms in the vicinity of the probe was calculated according to the procedure elaborated in appropriate literature [17, 33-35]. The results are presented in Fig. 8. A broad maximum is observed in each curve and the peak shifts gradually towards higher pressure as discharge power increases. Finally, the dissociation fraction of oxygen molecules in the remote chamber is calculated as

$\eta=\frac{n k T}{2 p_{1}}$.

Here $n$ is the O-atom density, $k$ is the Boltzmann constant, $T$ is the gas temperature in the remote chamber and $p_{1}$ is the pressure in the remote chamber. The temperature of the remote chamber remains at room temperature (approx. $300 \mathrm{~K}$ ). The dissociation fraction versus the pressure in the remote chamber is presented in Fig. 9.

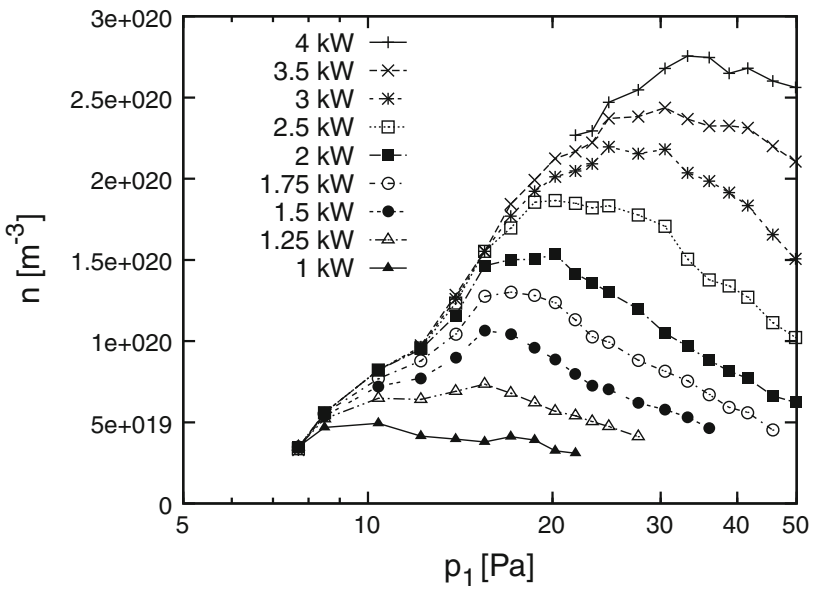

Fig. 8 The O-atom density versus pressure in the remote chamber. The discharge power is the parameter

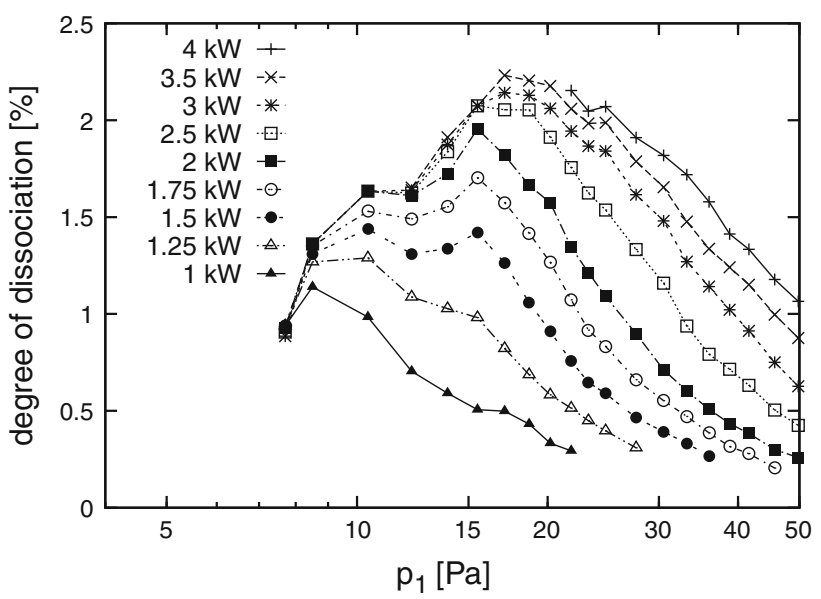

Fig. 9 The dissociation fraction of oxygen molecules versus pressure in the remote chamber

Some researchers prefer using a mixture of argon and oxygen from pure oxygen [36-39]. Specifically, the addition of argon often assures a higher dissociation fraction of oxygen molecules in the discharge itself, and helps prevent diffusion of atoms onto the walls where they might recombine. To check whether the addition of argon helps in this particular setup, we performed the following experiment. Another flow meter was installed to validate the applicability of this gas mixture; while the total flow was kept constant, the partial flows of oxygen and argon were varied from 0 to $100 \%$. The results are presented in Fig. 10 for two different pressures and two different discharge powers. The admixture of argon does not influence the $\mathrm{O}$-atom density much as long as the concentration of $\mathrm{Ar}$ is below about $50 \%$. As expected, the O-atom density in the remote chamber approaches zero when the argon concentration in the original gas mixture approaches $100 \%$.

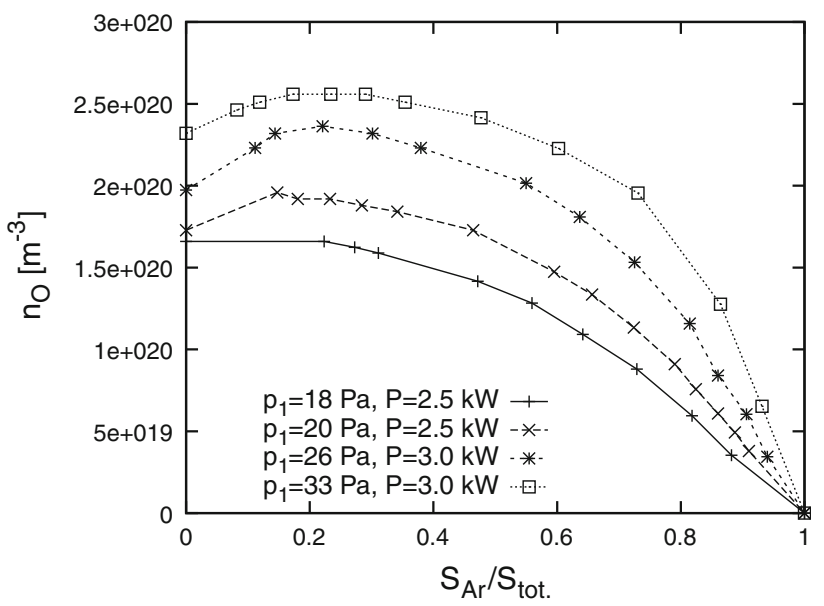

Fig. 10 The $\mathrm{O}$-atom density versus concentration of $\mathrm{Ar}$ in the gas mixture 
The density of oxygen atoms in the centre of the discharge tube cannot be measured in the present configuration. Still, it is needed to estimate the loss of atoms on their way from the discharge to the remote chamber. To estimate the $\mathrm{O}$ density in the discharge chamber a catalytic probe was mounted into a side tube of the discharge chamber as shown schematically in Fig. 1. The O density at the edge of the discharge chamber as determined from the measurements performed with this catalytic probe was measured for various discharge power settings at selected pressures. A comparison of results obtained in the discharge and remote chambers is plotted in Fig. 11. The ratio between the O-atom density at the edge of the discharge chamber and in the center of the remote chamber is plotted versus the effective pumping speed in Fig. 12.

It is worth mentioning that attempts were made to measure the O-atom density in the remote chamber while using only the rotary pump for pumping, but the measured

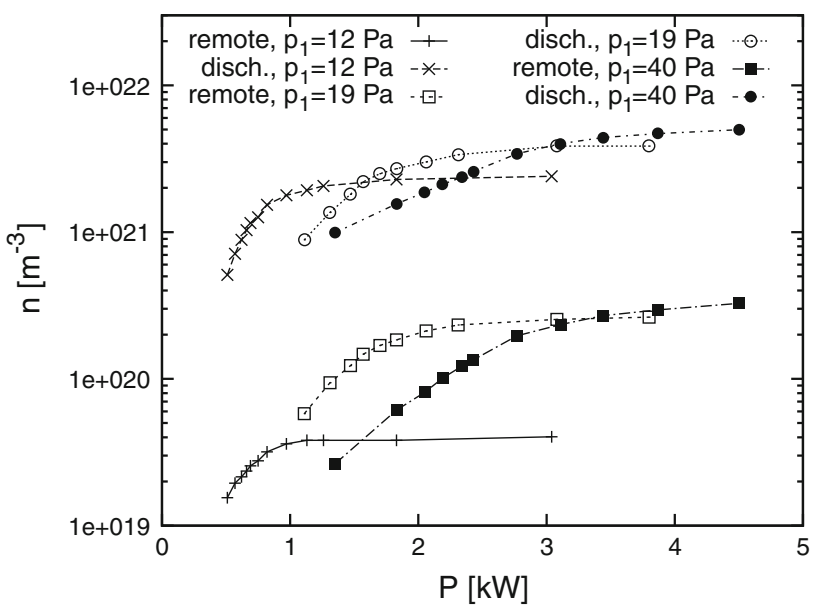

Fig. 11 The O-atom densities in discharge and remote chambers versus discharge power at selected pressures in the remote chamber

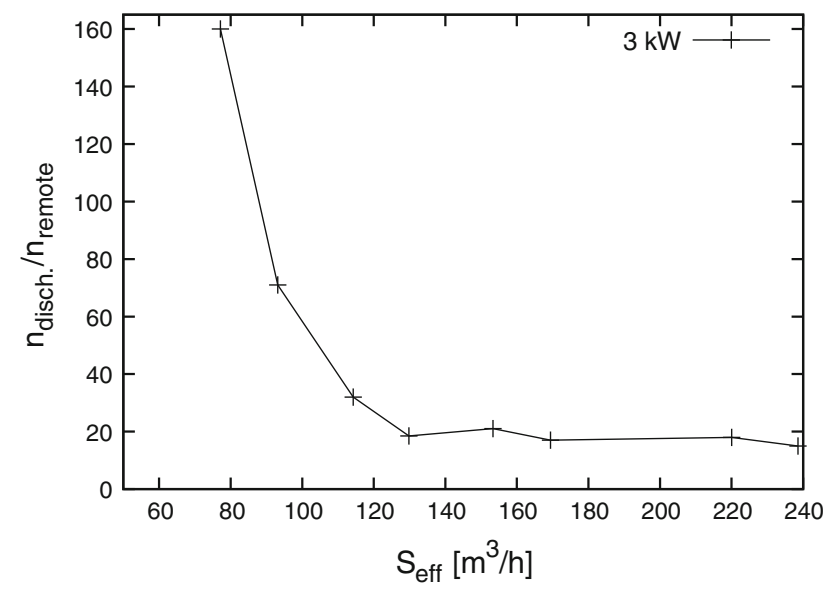

Fig. 12 The ratio between the O-atom density in the discharge and remote chambers at the power of $3 \mathrm{~kW}$

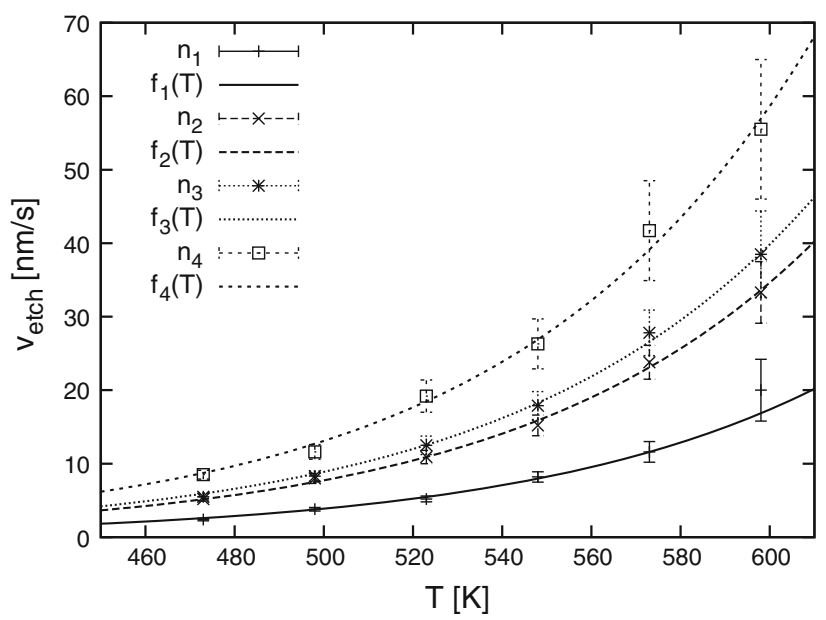

Fig. 13 Etching rate versus sample temperature at different oxygen atom densities with best fitted curves: $n_{1}=0.53 \times 10^{20} \mathrm{~m}^{-3}, f_{1}(T)=$ $2.14 \times 10^{-3} \mathrm{~nm} / \mathrm{s} e^{T / 66.7 \mathrm{~K}}, \quad n_{2}=1.1 \times 10^{20} \mathrm{~m}^{-3}, \quad f_{2}(T)=4.27 \times$ $10^{-3} \mathrm{~nm} / \mathrm{s} e^{T / 66.7 \mathrm{~K}}, n_{3}=1.3 \times 10^{20} \mathrm{~m}^{-3}, f_{3}(T)=4.92 \times 10^{-3} \mathrm{~nm} /$ $\mathrm{s} e^{T / 66.7 \mathrm{~K}}, n_{4}=1.84 \times 10^{20} \mathrm{~m}^{-3}, f_{4}(T)=7.24 \times 10^{-3} \mathrm{~nm} / \mathrm{s} e^{T / 66.7 \mathrm{~K}}$

signal was always close to the detection limit of our probe which presently is close to $1 \times 10^{19} \mathrm{~m}^{-3}$. The occurrence of such a low density under these pumping conditions will be explained in detail below.

The efficiency of hydrogenated carbon films removal has been tested in our experimental system. Thin films of hydrogenated carbon have been deposited onto well-polished stainless steel substrates by reactive sputtering of a graphite target in acetylene atmosphere. The thickness of the deposits was about $500 \mathrm{~nm}$. A heatable sample holder was installed into the remote chamber and samples were exposed to oxygen atoms. Figure 13 represents the measured etching rate of model hydrogenated carbon films versus temperature. The parameter in Fig. 13 is the oxygen atom density in the vicinity of samples. The measured data in Fig. 13 are fitted with exponential curves. The results indicate exponential relation between etching rate and samples temperature. In order to prove it, the curves are presented also in semilogaritmic diagram in Fig. 14. Figure 15 represents the same results but the parameter is now the sample temperature and the etching rate is presented versus the density of neutral O-atoms in the sample vicinity. Finally, the pre-exponential factor $a$ is plotted versus the density of neutral O-atoms in Fig. 16.

\section{Discussion}

The experimental results clearly show that the density of $\mathrm{O}$ atoms in the remote chamber is appreciably high providing the pumping speed is high enough. The absolute value is of the order of $10^{20} \mathrm{~m}^{-3}$. The resultant flux of atoms onto any 


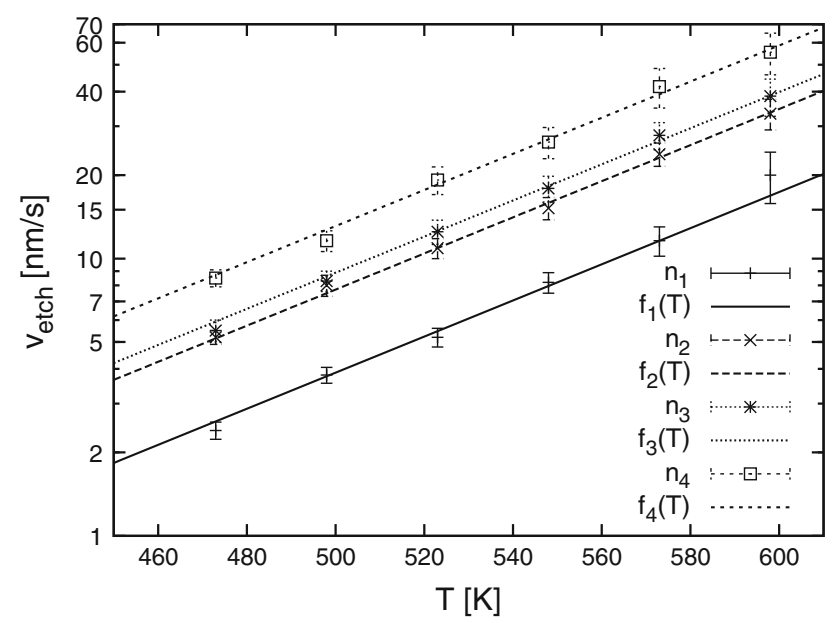

Fig. 14 Etching rate in logarithmic scale versus sample temperature at different oxygen atom densities with best fitted curves

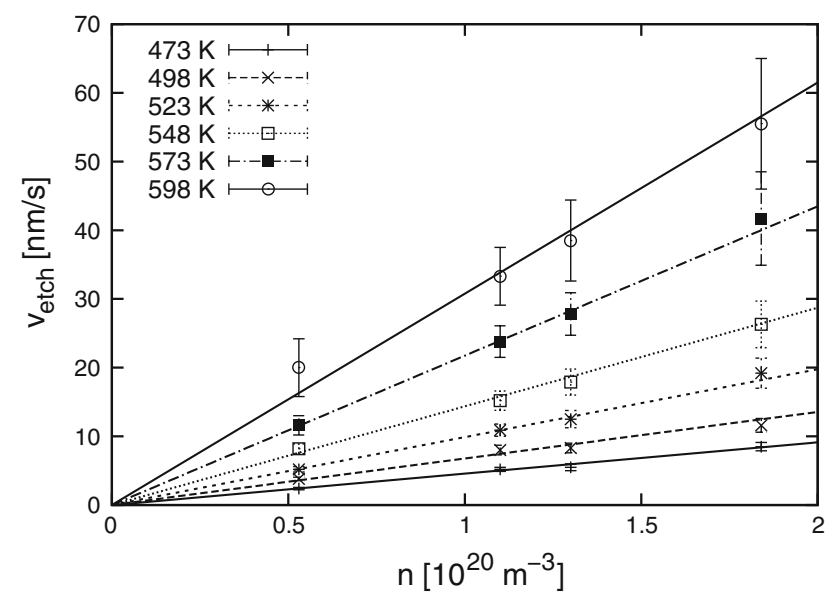

Fig. 15 Etching rate versus neutral oxygen atom densities at different sample temperatures with best fitted linear curves

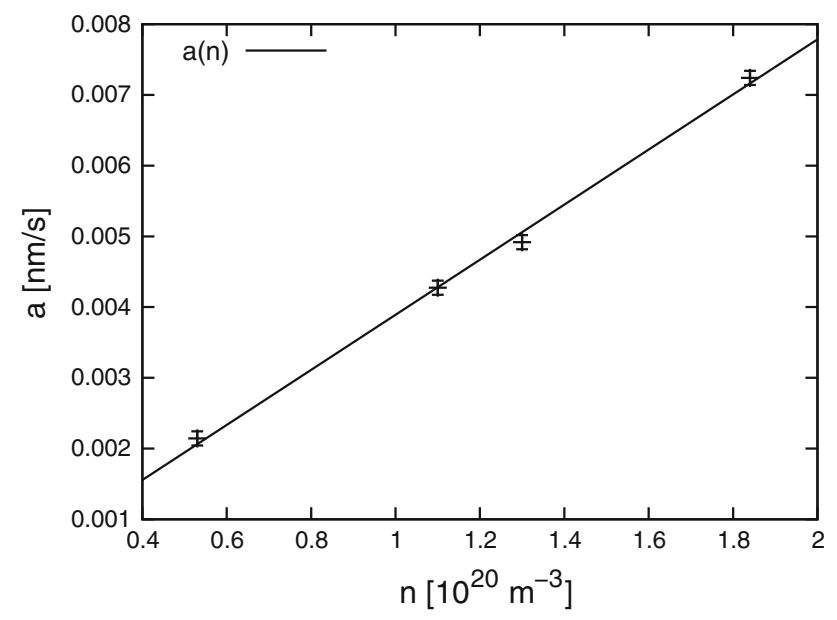

Fig. 16 Coefficient $a$ versus neutral oxygen atom densities with best fitted linear curve: $a(n)=3.91 \times 10^{-23} \mathrm{~nm} \mathrm{~s}^{-1} \mathrm{~m}^{3} n$ surface in the remote chamber is calculated using the standard equation

$j=\frac{n}{4} \bar{v}=\frac{n}{4} \sqrt{8 k T /(m \pi)}$.

Here $\bar{v}$ is the average random velocity of $\mathrm{O}$ atoms, $k$ the Boltzmann constant, $T$ is the gas temperature in the remote chamber, and $m$ is the mass of an oxygen atom. The temperature of the remote chamber remains at room temperature (approx. $300 \mathrm{~K}$ ) even after a long discharge operation, so the average velocity of oxygen atoms in this chamber is $630 \mathrm{~m} / \mathrm{s}$. The resultant flux of $\mathrm{O}$ atoms onto any surface in the remote chamber is therefore up to about $5 \times 10^{22} \mathrm{~m}^{-2} \mathrm{~s}^{-1}$. The surface area of the remote chamber is about $1 \mathrm{~m}^{2}$. The reasonably high flux of atoms and rather large surface area make the source of atoms suitable for treating large surfaces.

As mentioned above, the density of $\mathrm{O}$ atoms in the remote chamber was, while using only the rotary pump, immeasurable in practical terms. A crucial parameter is obviously the effective pumping speed. The influence of pumping speed is best observed in Fig. 12. The ratio between the $\mathrm{O}$-atom density at the edge of the discharge chamber and in the remote chamber decreases monotonically with increasing effective pumping speed. At low pumping speeds the ratio is higher than 100. Considering the results plotted in Fig. 12, the nominal pumping speed of the rotary pump is $80 \mathrm{~m}^{3} \mathrm{~h}^{-1}$, but if using just this pump the effective pumping speed is only as high as $30 \mathrm{~m}^{3} \mathrm{~h}^{-1}$ (see Fig. 5). Extrapolating the curve presented in Fig. 12 to the effective pumping speed $30 \mathrm{~m}^{3} \mathrm{~h}^{-1}$ would give a huge difference between the $\mathrm{O}$-atom density in the discharge and in the remote chamber.

An important conclusion can be drawn from Fig. 12; at high effective pumping speeds, the ratio between the O-atom density at the edge of the discharge chamber and the remote chamber stabilizes and approaches a constant value. For our particular experimental device, this constant value is approximately 20. Any further increase in the effective pumping speed beyond, say $150 \mathrm{~m}^{3} \mathrm{~h}^{-1}$, has practically no influence on this ratio. In practice, it means that for each configuration of the connecting tube, an appropriate pumping speed should be selected. There is no need to go to extremes - a reasonable pumping rate would do the job well. The effective pumping speeds in a tokamak are, of course, much higher than those obtained under our experimental conditions. This means that one could afford a much wider connecting tube in real experiments on tokamaks. Considering the conductance of the connecting tube (Fig. 6) and the required pumping speed (Fig. 12), one could estimate that the critical conditions for the optimal transfer of neutral oxygen atoms from the discharge chamber (placed away from the tokamak) to the remote 
areas of the tokamak where atoms should do their job (removal of hydrogenated carbon deposits) are met if the conductance of the connecting tube is close to the effective pumping speed. This practical consideration should be taken into account when constructing real experiments on tokamaks.

Let us now discuss the behavior of the O-atom density versus pressure and discharge power. The results of systematic measurements are presented in Fig. 8. The O-atom density at first increases with increasing pressure, reaches a maximum, and then decreases with further pressure increase. The increasing density at low pressure is merely an effect on increasing number of oxygen molecules available for dissociation. The number of molecules of course increases linearly with increasing pressure. In the region of increasing O-atom density, the density practically does not depend on the discharge power as long as it is reasonably high. In fact, a "general curve" is observed in Fig. 8. Measurements at any power follow this general curve but deviate from it at a certain pressure. The branching point depends on power, and the pressure at which branching is observed increases monotonously with increasing power. This result reveals an important rule; at a certain pressure, the $\mathrm{O}$-atom density does not depend much on power as long as it is high enough. Any increase in power in this regime has practically no effect on the $\mathrm{O}$ atoms density so one does not have to boost power as long as the pressure is low enough to assure for following the general curve.

As the pressure becomes sufficiently high, the O-atom density deviates from the general curve but quickly exhibits a maximum. The maximum shifts to higher pressure as the discharge power is increased. An important conclusion is that at a certain discharge power, an optimal pressure for the highest O-atom density exists. For example, at the power of $1.5 \mathrm{~kW}$ the optimal pressure is about $15 \mathrm{~Pa}$, at $3 \mathrm{~kW}$ it is almost $30 \mathrm{~Pa}$ while at $4 \mathrm{~kW}$ it is already $35 \mathrm{~Pa}$. To generalize, the higher the power is, the higher is the pressure required to obtain the maximum O-atom density. Such behavior is explained by the dissociation of oxygen molecules by electron impact in electrode-less RF discharges with predominant $\mathrm{H}$ mode. The electrons oscillate in the induced electric field inside the discharge coil. The maximum kinetic energy of the oscillating electrons increases with increasing discharge power. As long as the pressure is low enough, the mean free path of electrons is longer than the oscillation amplitude so the electrons gain the optimal kinetic energy from the induced electric field. At higher pressure, however, the electrons collide with neutral molecules before achieving the optimal energy so their heating is perturbed - they change their direction along the local electric field, so that heating is no longer particularly effective. This effect causes decreasing electron temperature with increasing pressure in the falling portions of the curves presented in Fig. 8. Obviously, a more intense electric field assures better acceleration of electrons and that explains the experimental observation of shifting the maximum of O-atom density towards higher pressure at increasing discharge power.

From upper limits, it is best to increase the discharge power to very high values to obtain the highest $\mathrm{O}$-atom density possible. In practice, however, there are limitations. Most of these arise from high voltages involved when running discharges at high power. It is well known that highfrequency electrode-less discharges are almost perfectly optimized for low powers [19-25]. The voltage on the RF coil is reasonable low, at a comfortably level of about $500 \mathrm{~V}$. Such systems are suitable for treatment of small samples. However, as mentioned in the Introduction $§ 1$, such small discharges are not suitable for large sources of atoms as required for removal of hydrogenated carbon deposits in tokamaks. Scaling up the discharges is not trivial requiring higher voltages to sustain plasmas in large discharge vessels. Voltages should be increased over a $\mathrm{kV}$ and this often causes huge problems. A higher voltage results in an increase in the capacitive coupling between the RF coil and any grounded metal objects in the vicinity, including the discharge vessel flanges. Not only the RF power is lost by this capacitive coupling but high-voltage sheaths appear as well. Positive ions are accelerated in such sheaths and cause bombardment of the plasma facing materials and thus sputtering. Sputtered material deposits anywhere in the discharge vessel and forms a layer of foreign material. Such a thin film has a higher coefficient for heterogeneous surface recombination of oxygen atoms than the originally smooth glass surface. This effect leads directly to a loss of $\mathrm{O}$ atoms on the surfaces and thus a poor dissociation fraction in the discharge vessel, also similarly reflected in the remote chamber.

Large powers should be therefore avoided. A method by which voltages are maintained at reasonable levels but still preserve plasma homogeneity in the system is the application of two or more parallel coils. This is why we used a double coil as described in the "Experimental Setup". The impedance of the coil (and the coil voltage) depends on the number of turns. A single-turn "coil", which is often used in small inductively coupled RF discharges operating in an almost pure $\mathrm{H}$ mode, has the lowest voltage but does not allow for creation of homogeneous plasma in large discharge tubes. As mentioned earlier, our discharge tube is two meters long. A reasonably uniform plasma at elevated pressure can only be created by using coils with many turns. Such coils, however, have high impedances and thus very high voltages appear. To minimize the high voltage effects but maintain a uniform plasma, we developed a two parallel coupled coil setup (see "Experimental Setup"). Such a configuration enables reasonable voltage levels to be achieved and a reasonable plasma uniformity. 
The dissociation fraction of oxygen molecules in the remote chamber is not great-an order of magnitude of $1 \%$ (see Fig. 9). Taking into account the ratio between the $\mathrm{O}$-atom density at the edge of the discharge and in the remote chamber (Fig. 12), the dissociation fraction in the remote chamber cannot be increased substantially by optimization of the $\mathrm{O}$ atom production in the discharge chamber. There is, however, a way of increasing the dissociation fraction (if one really insists on this) by using a mixture of oxygen and argon (or perhaps any other noble gas). The results presented in Fig. 10 clearly show that the dissociation fraction of oxygen molecules could be increased by a factor 1.5 or so solely by replacing about $40 \%$ of oxygen with argon. Otherwise, the dissociation fraction curves (Fig. 9) follow a qualitatively similar behavior as the $\mathrm{O}$-atom density in the remote chamber (Fig. 8) except that the maxima are shifted to somewhat lower pressures. The fact that the dissociation fraction decreases with decreasing pressure in the remote chamber could be attributed to a longer mean free path at lower pressures, a fast diffusion of atoms onto the walls of the connecting tube and the remote chamber, and thus more extensive loss by heterogeneous surface recombination. The recombination coefficient for glass and aluminum has been found to be very low [22, 40, 41] but still high enough to represent the major reason for the $\mathrm{O}$ atom loss on the way from the discharge to the remote chambers.

Let us finally discus the results of systematic removal of hydrogenated carbon deposits presented in Figs. 13-16. Measurements have been performed at different temperatures but measurably high values were only obtained at temperatures above $460 \mathrm{~K}$. The etching rate versus the temperature follows an exponential law:

$f(T)=a \cdot e^{b T}$.

Such behaviour is not unexpected since a chemical affinity for exothermic reactions often depends exponentially on the material temperature. It is encouraging that the etching rate of up to $50 \mathrm{~nm} / \mathrm{s}$ is obtained at the temperature of $570 \mathrm{~K}$ which is the most probable temperature of ITER during the first stage of operation. Systematic experiments performed at different temperatures and different densities of O-atoms allowed for statement of a general rule for removal of hydrogenated carbon deposit by neutral $\mathrm{O}$-atoms. The etching rate as a function of atom density and temperature is expressed as:

$v_{\text {etch }}(n, T)=a(n) \cdot e^{b T}$.

Since the curves presented in Fig. 15 are linear, the coefficient $a(n)$ in Eq. (8) can be replaced with a linear relation $a(n)=d \cdot n$ so the Eq. (8) reads as: $v_{\text {etch }}(n, T)=d \cdot n \cdot e^{b T}$.

The coefficients in (9) for our type of hydrogenated carbon deposit are determined from the results of systematic measurements presented in Figs. 13, 14, 15, and 16. The final form of the etching equation therefore reads as:

$v_{\text {etch }}(n, T)=3.91 \times 10^{-23} \frac{\mathrm{nm}}{\mathrm{s}} \mathrm{m}^{3} \cdot n \cdot e^{T / 66.7 \mathrm{~K}}$.

It is worth stressing that the coefficients definitely depend on the composition of deposits. In our case we addressed removal of rather hard deposits which are known to be difficult to etching by any method. Soft deposits rich hydrogen are probably etched at much higher rate. Namely our recent experiments on etching of polymers showed that the etching rate of the order of $10 \mathrm{~nm} / \mathrm{s}$ is obtained already at room temperature [42], where the etching rate of hydrogenated carbon deposits used at our experiments is only about $0.1 \mathrm{~nm} / \mathrm{s}$.

\section{Conclusions}

Systematic investigation on an oxygen plasma delivery system for a tokamak focusing on the density of $\mathrm{O}$ atoms in the remote chamber has produced important conclusions that are summarized as follows: (1) the O atoms can survive a long path from the discharge chamber to the remote chamber; (2) atom survival is unhindered despite the connecting tube having a number of sharp bends; (3) the most critical parameter governing survival is the effective pumping speed; (4) the optimal O-atom density in the remote chamber depends on the pressure and the discharge power-for each particular discharge power, an optimal pressure should be determined experimentally; (5) effective pumping speed needs not be excessive; (6) a high discharge power is needed to generate large quantities of $\mathrm{O}$ atoms and the problems arising from high voltages appearing at high powers could be minimized by using a specific coupling of RF coils and the generator; and (7) the dissociation fraction of $\mathrm{O}$ atoms in the remote chamber peaks at a pressure that depends on the discharge power, and can be increased by a factor of 1.5 using an appropriate mixture of argon and oxygen instead of pure oxygen. The results clearly show that, for removal of hydrogenated carbon deposits from remote parts of tokamaks with carbon materials present at the most exposed parts of divertors, a large source of $\mathrm{O}$ atoms can be suitably delivered for application in real reactor environments. Preliminary experiments with rather hard hydrogenated carbon deposits presented in this paper indicate that the removal rate at temperatures characteristic for operation of ITER in the first stage is several $10 \mathrm{~nm} / \mathrm{s}$ which is a suitable value for 
application of this technology in tokamaks with carbon divertors.

Acknowledgments The authors gratefully acknowledge the financial contribution by the European Fusion Development Association (EFDA) through contracts for basic and preferential support as well as the Slovenian Research Agency through contract nos. P2-0082. The operation was also financed in part by the European Union, European Social Fund.

Open Access This article is distributed under the terms of the Creative Commons Attribution License which permits any use, distribution, and reproduction in any medium, provided the original author(s) and the source are credited.

\section{References}

1. T. Loarer, J. Nucl. Mater. 390-391, 20 (2009)

2. F.L. Tabares, D. Tafalla, I. Tanarro, V.J. Herrero, A.M. Islyaikin, Vacuum 73, 161 (2004)

3. F.L. Tabares, D. Tafalla, V. Rohde, M. Stamp, G. Matthews, G. Esser, V. Philipps, R. Doerner, M. Baldwin, J. Nucl. Mater. 337-339, 867 (2005)

4. F.L. Tabares, V. Rohde, the ASDEX Upgrade Team, Nucl. Fusion 45, L27 (2005)

5. K. Sugiyama, Y. Sakawa, T. Tanabe, D. Watanabe, T. Shibahara, K. Masaki, J. Nucl. Mater. 405, 70 (2010)

6. J.W. Davis, A.A. Haasz, J. Nucl. Mater. 390-391, 532 (2009)

7. J.W. Davis, C.G. Hamilton, A.A. Haasz, R.G. Macaulay-Newcombe, J. Nucl. Mater. 305, 66 (2002)

8. J.W. Davis, A.A. Haasz, Phys. Scr. T91, 33 (2001)

9. T. Schwarz-Selinger, C. Hopf, C. Sun, W. Jacob, J. Nucl. Mater. 363-365, 174 (2007)

10. J.A. Ferreira, F.L. Tabares, D. Tafalla, J. Nucl. Mater. 363-365, 888 (2007)

11. A. Vesel, M. Mozetic, P. Panjan, N. Hauptman, M. KlanjsekGunde, M. Balat-Pichelin, Surf. Coat. Technol. 204, 1503 (2010)

12. W. Jacob, C. Hopf, M. Schlüter, Appl. Phys. Lett. 86, 204103 (2005)

13. A. Vesel, M. Mozetic, P. Panjan, N. Hauptman, M. KlanjsekGunde, M. Balat-Pichelin, Nucl. Eng. Des. 241, 1255 (2011)

14. V. Philipps, G. Sergienko, A. Lyssoivan, H.G. Esser, M. Freisinger, A. Kreter, U. Samm, J. Nucl. Mater. 363-365, 929 (2007)

15. C. Schulz, A. Kreter, V. Philipps, A. Litnovsky, U. Samm, J. Nucl. Mater. (in press)

16. N.V. Kholodkova, I.V. Kholodkov, I.N. Brovikova, High Temp. 47, 448 (2009)
17. G. Primc, R. Zaplotnik, A. Vesel, M. Mozetic, AIP Adv. 1, 022129 (2011)

18. T. Vrlinic, C. Mille, D. Debarnot, F. Poncin-Epaillard, Vacuum 83, 792 (2009)

19. M. Mozetic, A. Vesel, U. Cvelbar, A. Ricard, Plasma Chem. Plasma Process. 26, 103 (2006)

20. K. Kutasi, V. Guerra, P. Sa, J. Phys. D Appl. Phys. 43, 175201 (2010)

21. V. Guerra, K. Kutasi, P.A. Sa, Appl. Phys. Lett. 96, 071503 (2010)

22. A. Drenik, U. Cvelbar, A. Vesel, M. Mozetic, Inf. Midem 35, 85 (2005)

23. A. Vesel, M. Mozetic, Vacuum 61, 373 (2001)

24. I. Poberaj, M. Mozetic, D. Babic, J. Vac. Sci. Technol., A 20, 189 (2002)

25. M. Mozetic, Vacuum 71, 237 (2003)

26. J.E. Morgan, L. Ellias, H.I. Schiff, J. Chem. Phys. 33, 930 (1960)

27. D.S. Hacker, S.A. Marshall, M. Steinberg, J. Chem. Phys. 35, 1788 (1961)

28. S. Wickramanayataka, N. Hosokawa, Y. Hatanaka, Jpn. J. Appl. Phys. 30, 2897 (1991)

29. J.C. Greaves, J.W. Linnett, Trans. Faraday Soc. 55, 1346 (1959)

30. A. Drenik, A. Vesel, M. Mozetic, J. Nucl. Mater. 386-388, 893 (2009)

31. A. Drenik, K. Elersic, M. Modic, P. Panjan, Mater. Tehnol. 45, $281(2011)$

32. W. Umrath, Fundamentals of Vacuum Technology (Oerlikon Leybold, Cologne, 2007), pp. 16-17

33. M. Balat-Pichelin, M. Pissarelli, A. Vesel, Mater. Chem. Phys. 123, 40 (2010)

34. M. Balat-Pichelin, A. Vesel, Chem. Phys. 327, 112 (2006)

35. A. Vesel, M. Mozetic, M. Balat-Pichelin, Vacuum 81, 1088 (2007)

36. U. Cvelbar, M. Mozetic, D. Babic, I. Poberaj, A. Ricard, Vacuum 80, 904 (2006)

37. K. Takechi, M.A. Lieberman, J. Appl. Phys. 90, 3205 (2001)

38. H.C. Baxter, G.A. Gaynor, P.R. Richardson, A.C. Jones, I.R. Whittle et al., IEEE Trans. Plasma Sci. 34, 1337 (2006)

39. M. Tadokoro, H. Hirata, N. Nakano, Z.L. Petrović, Z. Makabe, Phys. Rev. E 57, R43 (1998)

40. A. Drenik, U. Cvelbar, A. Vesel, M. Mozetic, Strojarstvo 48, 17 (2006)

41. A. Drenik, Probability of Heterogeneous Recombination of Hydrogen and Oxygen Atoms on the Surface of Fusion-Relevant Materials. PhD Thesis (Jozef Stefan Inst. Postgraduate School, Ljubljana, 2009), p. 101

42. A. Doliska, A. Vesel, M. Kolar, K. Stana-Kleinschek, M. Mozetic, Surf. Interface Anal. 44, 56 (2012) 